\title{
Primera parte: Almacenamiento insaturado de agua en un perfil de suelo
}

\section{First part: Storage unsaturated water in a soil profile}

Adrián Enrique Chavarría-Vidal ${ }^{1}$

Chavarría-Vidal, A.E. Primera parte: Almacenamiento insaturado de agua en un perfil de suelo. Tecnología en Marcha.

Vol. 34-3, Julio-Setiembre 2021. Pág 15-33.

doi https://doi.org/10.18845/tm.v34i3.5009 


\title{
Palabras clave
}

Almacenamiento del agua en el suelo; recurso hídrico; suelo insaturado; humedad del suelo para las plantas.

\section{Resumen}

Un fundamento para la correcta y eficiente aplicación y uso del recurso hídrico en los sistemas de producción agrícola es conocer el estado de humedad en que se encuentra el suelo ya que la cantidad a aplicar debe ser la misma que la cantidad consumida en el sistema suelo - planta por medio de la evapotranspiración. Este estado de humedad se debe de estimar lo mayor cercano posible a la realidad y para ello se debe de tomar el perfil del suelo a una profundidad a la cual se adapte lo mejor posible al o a los cultivos presentes. Normalmente la profundidad adecuada se refiere a la profundidad de raíces de las plantas y la forma de estimación se puede dar de varias maneras y una de ellas es por medio de la humedad volumétrica la cual, se puede estimar de manera directa como indirecta (como ejemplo podemos mencionar sensores calibrados). No obstante, con la información obtenida del campo se debe de realizar la estimación total respectiva que va a depender de la metodología utilizada respecto a la profundidad definida, el número de muestreos y la distancia entre los muestreos, los cuales; estos últimos se recomienda que sean de la misma longitud.

Por ello el objetivo fundamental de este artículo es presentar diferentes opciones de manejo de la información obtenida de campo para estimar los contenidos hídricos insaturados en un perfil del suelo, compararlos en sus estimaciones para la toma de mejores decisiones.

\section{Keywords}

Water storage in the ground; hidric resource; unsaturated soil; soil moisture for plants.

\begin{abstract}
A foundation for the correct and efficient application and use of the water resource in agricultural production systems is to know the state of humidity in which the soil is found since the amount to be applied must be the same as the amount consumed in the soil system - plant through evapotranspiration. This state of humidity must be estimated as close as possible to reality and for this, the soil profile should be taken at a depth to which it adapts best to the crop (s) present. Normally the appropriate depth refers to the depth of the roots of the plants and the form of estimation can be given in several ways and one of them is by means of the volumetric humidity which, can be estimated directly as indirectly (as an example we can mention calibrated sensors). However, with the information obtained from the field, the respective total estimate must be made and will depend on the methodology used with respect to the defined depth, the number of samples and the distance between the samples, which; The latter are recommended to be the same length. Therefore, the main objective of this article is to present different options for managing the information obtained from the field to estimate unsaturated water contents in a soil profile, compare them in their estimates for better decision making.
\end{abstract}




\section{Introducción}

La estimación del almacenamiento del agua en el suelo está revestido de alta importancia para la planeación y gestión del recurso hídrico orientado al riego de los cultivos de ahí la urgencia de masificar la cultura del buen aprovechamiento del agua con el objetivo de asegurar el suministro alimentario y para ello se recomienda la utilización de cultivos de enraizamiento profundo que resisten más a la sequias [1].

El suelo es el depósito de almacenamiento de agua, aire y nutrientes desde donde las plantas los extraen. La capacidad de almacenamiento y la disponibilidad para las plantas depende de las cantidades existentes y de las características de cada suelo. Por lo tanto es necesario conocer cómo están constituidos los suelos y las fuerzas que actúan en la retención y movimiento del agua [2].

El suelo juega un rol importante en el manejo del agua de riego, ya que es el "macetero natural" que tienen las raíces para absorber el agua de riego o lluvia efectiva. La cantidad de agua que puede retener un suelo depende de sus propiedades físicas, dada por la textura, estructura, contenido de materia orgánica $(\mathrm{MO})$, profundidad, entre otras. La textura se encuentra representada por el porcentaje de arena, limo y arcilla. Un suelo presenta una buena textura cuando los porcentajes de estos elementos (arena, limo y arcilla) en su conjunto le entregan a la planta un soporte para un buen desarrollo radicular y un adecuado nivel nutricional [3].

El agua que precipita en forma de lluvia puede quedar depositada en la superficie vegetal, alcanzar la superficie del suelo e infiltrarse o alcanzar la superficie del suelo y escurrir o alcanzar la superficie del suelo e infiltrar y escurrir. El volumen de agua almacenado disponible para las plantas es aquel que, a pesar de estar en movimiento permanece en la zona radical del cultivo el tiempo suficiente para ser absorbido por las plantas. En este aspecto, los microporos del suelo cumplen una función importante, al mantener el agua por más tiempo disponible para las plantas [4].

El almacenamiento del agua en el suelo insaturado se encuentra en función de la profundidad a la que se quiere conocer dicho almacenamiento y para su cálculo se conocen dos reglas que son la regla del Trapecio o Trapezoidal y la regla de Simpson [5] [6] [7]. Para calcular la cantidad de agua almacenada en el perfil del suelo se debe de integrar las medidas de los contenidos de agua desde la superficie hasta la profundidad deseada la cual está representada por la profundidad de raíces del cultivo [8], fundamentalmente por aquella profundidad de raíces efectiva en la absorción de agua conjuntamente con los nutrientes diluidos; por lo que se presentan errores en las determinaciones de la humedad del suelo y al método de integración [9] [10].

\section{Aspectos metodológicos}

Las ecuaciones de la 1 a la 4 que se verán demostradas más adelante en el documento fueron tomadas del libro Apuntes de clases de Relación Suelo Agua Planta como fundamento y las otras ecuaciones de almacenamiento del agua en el suelo fueron desarrolladas respectivamente. No obstante, se desarrollan ejemplos para realizar las respectivas comparaciones de exactitud y precisión.

\section{Desarrollo}

Para medir humedad del suelo existen diferentes maneras tanto directas como indirectas y una de ellas es mediante el concepto expresado en las siguientes ecuaciones 1 y 2 [11]: 
ec. 1:

$$
h(m m)=\frac{\% \theta v}{100} * \mathrm{Ps}
$$

o ec. 2:

$$
h(m m)=\theta v * \mathrm{Ps}
$$

donde

$\% \theta v$ : porcentaje de humedad volumétrica en el suelo

$\theta v$ : humedad volumétrica en el suelo en proporción con base a la unidad

Ps: profundidad del suelo o grosor de un segmento del perfil del suelo $(\mathrm{mm})$

$h$ : cantidad de agua existente en un estrato de suelo a una profundidad del perfil del suelo expresado en lámina de agua $(\mathrm{mm})$. La $h$ puede presentarse en "mm" o en "cm" pero se recomienda mayormente expresarlo en las unidades de " $m m$ ".

Para expresar dicha humedad en metros cúbicos " $\mathrm{m}^{3}$ " por cada hectárea (ha) se puede utilizar la siguiente ecuación 3 y 4 :

ec 3:

$$
V(m 3 / h a)=h(m m) * 10=\left(\frac{\% \theta v}{100} * \mathrm{Ps}\right) * 10
$$

ec. 4:

$$
V(m 3 / h a)=h(m m) * 10=\theta v * \text { Ps } * 10
$$

donde

$V$ : cantidad de agua existente en un estrato de suelo con un grosor dado a una profundidad del perfil del suelo expresado en un volumen de agua por área $\left(\mathrm{m}^{3} / \mathrm{ha}\right)$.

$\% \theta v=\% \theta$ : porcentaje de humedad volumétrica en el suelo

$\theta v=\theta$ : humedad volumétrica en el suelo en proporción con base a la unidad

Ps: profundidad del suelo o de un segmento del suelo ( $\mathrm{mm}$ )

Debido a que en el suelo se presentan diferentes cantidades o variaciones en el contenido de humedad con respecto a la variación de la profundidad del mismo, se ha tomado usualmente, como una metodología aceptada para estimar dicha humedad, subdividir la profundidad en segmentos de igual longitud cada uno para mejorar el estimado de la cantidad de humedad presente en el suelo, claro está; teniendo la obligación de medir la humedad de cada segmento. Como ejemplo de ello es tomar una profundidad de suelo de un metro y subdividirla en 10 segmentos de $10 \mathrm{~cm}$ cada una o en su defecto, segmentos de $20 \mathrm{~cm}$ para un total de 5 en 1,0 $\mathrm{m}$ de profundidad. A menor distancia del segmento se aumenta la exactitud de la estimación cuyo objetivo es tener un mayor acercamiento a la cantidad real del almacenamiento de agua que existe en un perfil de suelo a una profundidad específica, pero aumenta también el número de segmentos y como tal, el número de muestras para medir la humedad de cada segmento. 
Esta metodología presenta el inconveniente de que no toma en cuenta los diferentes horizontes presentes en el suelo lo cual, frecuentemente manifiestan diferentes texturas, estructuras, distribución, tamaño y continuidad del espacio poroso y otros.

Lo anterior provoca que esta metodología presente cierta impresión ante suelos con anisotropía textural, pero ante suelos uniformes en profundidad o con pocas variaciones esta metodología se fortalece presentando mayor exactitud y precisión por lo cual se obtienen valores más cercanos a los reales con respecto al contenido de humedad del suelo. No obstante, el error tiende a disminuir cuando la distancia de cada segmento tiende a ser menor.

Para las estimaciones con esta metodología se puede utilizar tanto la regla del trapecio como la regla de la función cuadrática. En nuestro caso utilizaremos la regla del trapecio.

Las formas para realizar la medida y la estimación en profundidad de la humedad en un perfil de suelo son las siguientes:

\section{Metodología 1}

Estimación de la humedad del suelo con contenido de agua no saturado en su perfil dividido en segmentos iguales en su profundidad $(\Delta Z$ ) (no se mide la humedad superficial del suelo) y su respectiva humedad volumétrica ( $\theta v)$ para cada segmento, donde se comienza a medir la humedad a partir del primer medio segmento $(\Delta Z / 2)$ y luego se continúa la medición de la humedad en profundidad a la longitud definida del segmento $(\triangle Z)$.

Tomando en cuenta la figura 1, la fórmula para estimar el agua que se encuentra almacenada en el suelo en un momento dado se demuestra de la siguiente manera:

Humedad volumétrica en profundidad del perfil del suelo $\theta$

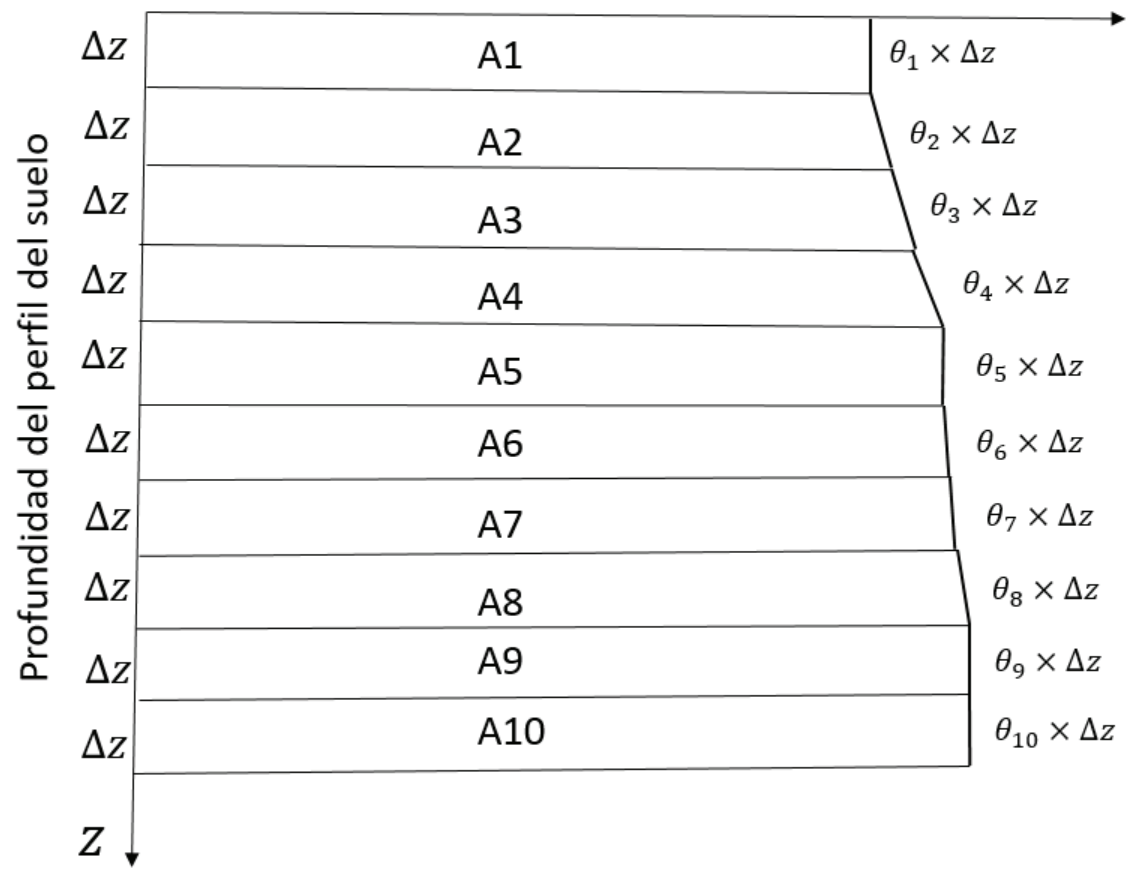

Figura 1. Estimación de humedad del suelo realizando la primera medida a partir del primer $\Delta z / 2$ y luego cada $\Delta z$ en profundidad 


$$
\begin{gathered}
h(m m)=\Delta Z * \theta 1+\Delta Z * \theta 2+\Delta Z * \theta 3+\Delta Z * \theta 4+\Delta Z * \theta 5+\Delta Z * \theta 6+\Delta Z * \theta 7+\Delta Z \\
* \theta 8+\Delta Z * \theta 9+\Delta Z * \theta 10 \\
h(m m)=\Delta Z *(\theta 1+\theta 2+\theta 3+\theta 4+\theta 5+\theta 6+\theta 7+\theta 8+\theta 9+\theta 10)
\end{gathered}
$$

donde sustituyendo por se puede deducir la ecuación 5

ec. 5:

$$
h(m m)=\Delta Z * \sum_{i=1}^{n} \theta i
$$

y para el caso en que la humedad volumétrica se encuentre en términos de porcentaje tenemos la ecuación 6:

ec. 6:

$$
h(m m)=\Delta Z * \sum_{i=1}^{n} \frac{\% \theta i}{100}
$$

donde:

$\Delta Z=$ longitud del segmento de profundidad del suelo $(\mathrm{mm})$

$n=$ número total de capas en el perfil del suelo

$\theta i=$ humedad del suelo volumétrica dada en proporción con base a la unidad, que se presenta en cada uno de los segmentos

$\% \theta i=$ porcentaje de humedad volumétrica del suelo que se presenta en cada uno de los segmentos

Para expresar dicha humedad en metros cúbicos por cada hectárea se puede utilizar la siguiente ecuación 7:

ec. 7:

$$
h\left(\frac{m 3}{h a}\right)=\left(\Delta Z * \sum_{i=1}^{n} \theta i\right) * 10
$$

y para el caso en que la humedad volumétrica se encuentre en términos de porcentaje tenemos la ecuación 8:

ec. 8:

$$
h\left(\frac{m 3}{h a}\right)=\left(\Delta Z * \sum_{i=1}^{n} \frac{\% \theta i}{100}\right) * 10
$$

Ejemplo 1: Se tiene un perfil de suelo de 1,0 m de profundidad al cual se le hicieron muestreos para medir la humedad que hay en él. Para ello se dividió el perfil en capas de $20 \mathrm{~cm}$ donde cada una representa cada segmento $\Delta z$ en profundidad y la humedad volumétrica se midió en el centro de cada capa o segmento $\Delta z$. Para dicha medida se empezó a partir de los primeros 
$10 \mathrm{~cm}$ que representa el primer $\Delta \mathrm{z} / 2$, pero luego se continuó con las medidas de humedad cada $20 \mathrm{~cm}$ como se muestra en el cuadro 1. Calcular el total de la humedad retenida en el perfil del suelo según metodología 1.

Cuadro 1. Valores que describen tanto la forma de muestreo como los valores de humedad obtenidos en el suelo empezando la medida de humedad a los $10 \mathrm{~cm}$ de profundidad.

\begin{tabular}{|c|c|c|}
\hline $\begin{array}{c}\text { Profundidad de } \\
\text { cada segmento } \\
(\mathrm{cm})\end{array}$ & $\begin{array}{c}\text { Profundidad de } \\
\text { la medida de } \\
\text { humedad en el } \\
\text { suelo }(\mathrm{cm})\end{array}$ & $\begin{array}{c}\text { Humedad } \\
(\% \mathrm{HV})\end{array}$ \\
\hline 0 & - & - \\
\hline 20 & 10 & 16 \\
\hline 40 & 30 & 19 \\
\hline 60 & 50 & 21 \\
\hline 80 & 70 & 27 \\
\hline 100 & 90 & 38 \\
\hline
\end{tabular}

Utilizando la ecuación 6

$\Delta Z=20 \mathrm{~cm}=200 \mathrm{~mm}$

$0,5 * \Delta Z=10 \mathrm{~cm}=100 \mathrm{~mm}$

$$
\begin{gathered}
h=\Delta Z * \sum_{i=1}^{n} \frac{\% \theta i}{100} \\
h(\mathrm{~mm})=\frac{200 *(16+19+21+27+38)}{100} \\
h(\mathrm{~mm})=\frac{200 *(121)}{100} \\
h(\mathrm{~mm})=\frac{24200}{100} \\
h(\mathrm{~mm})=242
\end{gathered}
$$

Para conocer el total de agua que se encuentra en una hectárea a 1,0 $\mathrm{m}$ de profundidad utilizando la ecuación 3 tenemos:

$$
\begin{gathered}
h\left(\frac{m 3}{h a}\right)=242 * 10 \\
h\left(\frac{m 3}{h a}\right)=2420
\end{gathered}
$$


Como tal se puede observar que con la información de campo se estimó que a una profundidad de $1,0 \mathrm{~m}$ y en 1 ha se tienen $2420 \mathrm{~m}^{3}$.

\section{Metodología 2}

Estimación de la humedad del suelo con contenido de agua no saturado en su perfil dividido en segmentos iguales en su longitud $(\Delta Z$ ) (no se mide la humedad superficial del suelo) y su respectiva humedad volumétrica $(\theta \mathrm{v})$ para cada segmento, donde se comienza a medir la humedad a partir del primer segmento $(\Delta Z)$ y luego se continúa con la medición de la humedad en profundidad a la longitud definida del segmento $(\Delta Z)$. En esta metodología se supone que la humedad superficial es igual a la humedad que se encuentra a la primera profundidad $\Delta Z$.

Tomando en cuenta la figura 2, la fórmula para estimar el agua que se encuentra almacenada en el suelo en un momento dado se demuestra de la siguiente manera:

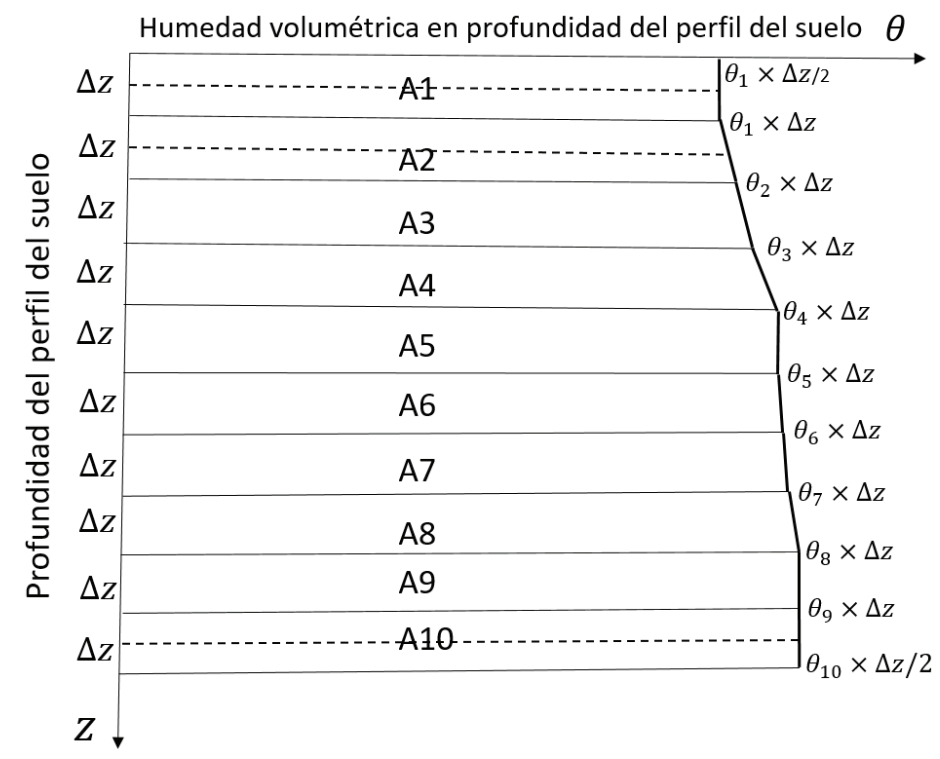

Figura 2. Estimación de humedad del suelo realizando la primera medida a partir del primer $\Delta z$ y luego cada $\Delta z$ en profundidad.

$$
\begin{gathered}
h(m m)=0,5 * \theta 1 * \Delta Z+\theta 1 * \Delta Z+\theta 2 * \Delta Z+\theta 3 * \Delta Z+\theta 4 * \Delta Z+\theta 5 * \Delta Z+\theta 6 * \Delta Z \\
+\theta 7 * \Delta Z+\theta 8 * \Delta Z+\theta 9 * \Delta Z+0,5 * \theta 10 * \Delta Z
\end{gathered}
$$

$$
h(m m)=(1,5 * \theta 1 * \Delta Z)+\left(\Delta Z * \sum_{i=2}^{n-1} \theta i\right)+(0,5 * \theta 10 * \Delta Z)
$$

sustituyendo $\theta 10$ por $\theta n$ se puede deducir la ecuación 9:

$$
h(m m)=(1,5 * \Delta Z * \theta 1)+(0,5 * \theta n * \Delta Z)+\left(\Delta Z * \sum_{i=2}^{n-1} \theta i\right)
$$

ec. 9:

$$
h(m m)=\Delta Z *\left(1,5 * \theta 1+0,5 * \theta n+\sum_{i=2}^{n-1} \theta i\right)
$$


y para el caso donde la humedad volumétrica esté expresada en porcentaje tenemos la ecuación 10

ec. 10:

$$
h(m m)=\Delta Z *\left(1,5 * \frac{\% \theta 1}{100}+0,5 * \frac{\% \theta n}{100}+\sum_{i=2}^{n-1} \frac{\% \theta i}{100}\right)
$$

donde:

$h=$ cantidad de agua en el perfil del suelo $(\mathrm{mm})$

$\Delta Z=$ longitud del segmento de profundidad del suelo $(\mathrm{mm})$

$n=$ número total de capas o de segmentos en el perfil del suelo

$\theta 1$ = humedad del suelo volumétrica medida al primer $\Delta Z$ de profundidad dada en proporción con base a la unidad

$\theta i=$ humedad del suelo volumétrica dada en proporción con base a la unidad, que se presenta en cada uno de los segmentos

$\theta n=$ humedad del suelo volumétrica dada en proporción con base a la unidad, que se presenta en el último segmento

$\% \theta 1=$ porcentaje de humedad volumétrica del suelo medida a la profundidad del primer $\Delta Z$

$\% \theta i$ = porcentaje de humedad volumétrica del suelo que se presenta en cada uno de los segmentos

$\% \theta n=$ porcentaje de humedad volumétrica del suelo que se presenta en el último segmento

Ejemplo 2: Se tiene un perfil de suelo de 1,0 m de profundidad al cual se le hicieron muestreos para medir la humedad que hay en él. Para ello se dividió el perfil en capas de $20 \mathrm{~cm}$ donde cada una representa cada $\Delta z$ en profundidad y la humedad volumétrica se midió a partir de cada capa $\Delta z$ por lo que se empezó a partir de los primeros $20 \mathrm{~cm}$, y luego se continuó con las medidas de humedad cada $20 \mathrm{~cm}$. Se toma la información del cuadro 2. Calcular el total de la humedad retenida en el perfil del suelo según metodología 2.

Cuadro 2. Valores que describen tanto la forma de muestreo como los valores de humedad obtenidos en el suelo empezando la medida de humedad a los $20 \mathrm{~cm}$ de profundidad

\begin{tabular}{|c|c|c|}
\hline $\begin{array}{c}\text { Profundidad de } \\
\text { cada segmento } \\
(\mathrm{cm})\end{array}$ & $\begin{array}{c}\text { Profundidad de la } \\
\text { medida de humedad } \\
\text { en el suelo (cm) }\end{array}$ & $\begin{array}{c}\text { Humedad } \\
(\% \mathrm{HV})\end{array}$ \\
\hline 0 & - & - \\
\hline 20 & 20 & 16 \\
\hline 40 & 40 & 19 \\
\hline 60 & 60 & 21 \\
\hline 80 & 80 & 27 \\
\hline 100 & 100 & 38 \\
\hline
\end{tabular}


Utilizando la ecuación 10:

$$
\begin{gathered}
h(\mathrm{~mm})=\Delta Z *\left(1,5 * \frac{\% \theta 1}{100}+0,5 * \frac{\% \theta n}{100}+\sum_{i=2}^{n-1} \frac{\% \theta i}{100}\right) \\
h(\mathrm{~mm})=200 *\left(1,5 * \frac{16}{100}+0,5 * \frac{38}{100}+\frac{19+21+27}{100}\right) \\
h(\mathrm{~mm})=200 * 1.1 \\
h(\mathrm{~mm})=220
\end{gathered}
$$

Para conocer el total de agua que se encuentra en una hectárea a 1,0 $\mathrm{m}$ de profundidad utilizando la ecuación 3 tenemos:

$$
\begin{gathered}
h\left(\frac{m 3}{h a}\right)=220 * 10 \\
h\left(\frac{m 3}{h a}\right)=2200
\end{gathered}
$$

\section{Metodología 3}

Estimación de la humedad del suelo con contenido de agua no saturado en su perfil dividido en segmentos iguales en su longitud $(\Delta Z)$ y su respectiva humedad volumétrica $(\theta v)$ para cada segmento, donde se comienza a medir la humedad a partir a partir de la superficie del suelo $(\theta 0)$ y luego continuando la medición a la longitud definida del segmento $(\Delta Z)$. En esta metodología 3 se supone que la humedad superficial es para el primer $\Delta Z / 2$.

Tomando en cuenta la figura 3, la fórmula para estimar el agua que se encuentra almacenada en el suelo en un momento dado se demuestra de la siguiente manera:

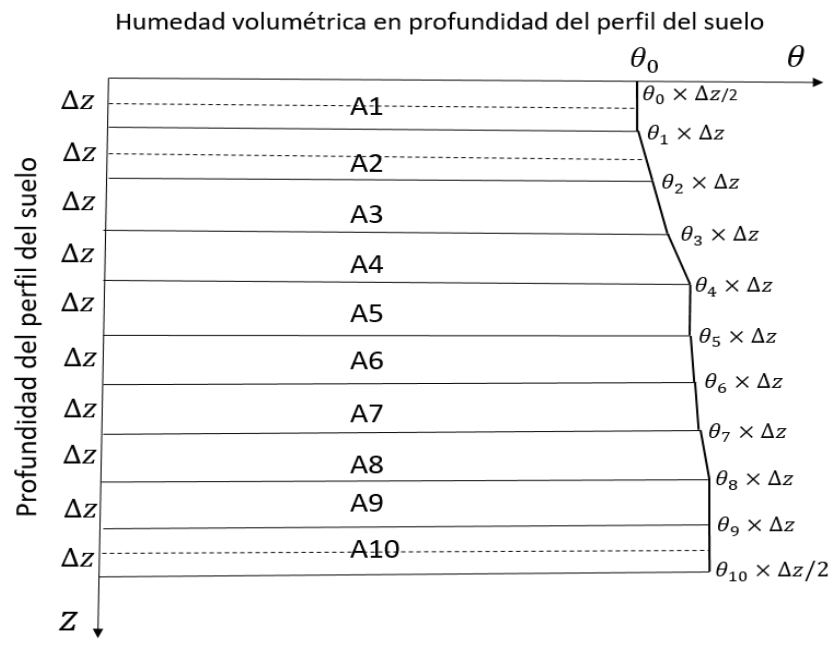

Figura 3. Estimación de la humedad del suelo realizando la primera medida a partir de la superficie del suelo $(\Delta z=0)$ y luego cada $\Delta z$ en profundidad 


$$
\begin{gathered}
h(\mathrm{~mm})=\theta o * 0,5 * \Delta Z+\theta 1 * \Delta Z+\theta 2 * \Delta Z+\theta 3 * \Delta Z+\theta 4 * \Delta Z+\theta 5 * \Delta Z+\theta 6 * \Delta Z \\
+\theta 7 * \Delta Z+\theta 8 * \Delta Z+\theta 9 * \Delta Z+\theta 10 * 0,5 * \Delta Z \\
h(\mathrm{~mm})=(0,5 * \theta o * \Delta Z)+\left(\Delta Z * \sum_{i=1}^{n-1} \theta i\right)+(0,5 * \theta 10 * \Delta Z) \\
h(\mathrm{~mm})=(0,5 * \theta o * \Delta Z)+(0,5 * \theta 10 * \Delta Z)+\left(\Delta Z * \sum_{i=1}^{n-1} \theta i\right)
\end{gathered}
$$

sustituyendo $\theta 10$ por $\theta n$ y factorizando podemos deducir la ecuación 11:

$$
h(m m)=0,5 * \Delta Z *(\theta o+\theta n)+\left(\Delta Z * \sum_{i=1}^{n-1} \theta i\right)
$$

ec. 11:

$$
h(m m)=\Delta Z *\left[0,5 *(\theta o+\theta n)+\sum_{i=1}^{n-1} \theta i\right]
$$

y para el caso donde la humedad volumétrica esté expresada en porcentaje tenemos la ecuación 12

ec. 12:

$$
h(m m)=\Delta Z *\left[0,5 *\left(\frac{\% \theta o+\% \theta n}{100}\right)+\sum_{i=1}^{n-1} \frac{\% \theta i}{100}\right]
$$

donde:

$\Delta Z=$ longitud del segmento de profundidad del suelo $(\mathrm{mm})$

$n=$ número total de capas o segmentos en el perfil del suelo

$\theta o=$ humedad del suelo volumétrica superficial dada en proporción con base a la unidad $\theta i=$ humedad del suelo volumétrica dada en proporción con base a la unidad, que se presenta en cada uno de los segmentos

$\theta n=$ humedad del suelo volumétrica dada en proporción con base a la unidad, que se presenta en el último segmento

$\% \theta 0$ = porcentaje de humedad volumétrica del suelo a nivel superficial

$\% \theta i=$ porcentaje de humedad volumétrica del suelo que se presenta en cada uno de los segmentos

$\% \theta n=$ porcentaje de humedad volumétrica del suelo que se presenta en el último segmento 
Ejemplo 3: Se tiene un perfil de suelo de 1,0 m de profundidad al cual se le hicieron muestreos para medir la humedad que hay en él. Para ello se dividió el perfil en capas de $20 \mathrm{~cm}$ donde cada una representa cada $\Delta z$ en profundidad y la humedad volumétrica se midió a partir de la superficie del suelo y luego se continuó con las medidas de humedad cada $20 \mathrm{~cm}$ o cada segmento $\Delta z$. Se toma la información del cuadro 2. Calcular el total de la humedad retenida en el perfil del suelo según metodología 3.

Cuadro 3. Valores que describen tanto la forma de muestreo como los valores de humedad en el suelo empezando el muestreo a 105 0,0 cm de profundidad.

\begin{tabular}{|c|c|c|}
\hline $\begin{array}{c}\text { Profundidad de } \\
\text { cada segmento } \\
(\mathrm{cm})\end{array}$ & $\begin{array}{c}\text { Profundidad de la } \\
\text { medida de humedad } \\
\text { en el suelo }(\mathrm{cm})\end{array}$ & $\begin{array}{c}\text { Humedad } \\
(\% \mathrm{HV})\end{array}$ \\
\hline 0 & 0 & 8 \\
\hline 20 & 10 & 16 \\
\hline 40 & 30 & 19 \\
\hline 60 & 50 & 21 \\
\hline 80 & 70 & 27 \\
\hline 100 & 90 & 38 \\
\hline
\end{tabular}

Utilizando la ecuación 12 tenemos:

$$
\begin{gathered}
h(\mathrm{~mm})=\Delta Z *\left[0,5 *\left(\frac{\% \theta o+\% \theta n}{100}\right)+\sum_{i=1}^{n-1} \frac{\% \theta i}{100 i}\right] \\
h(\mathrm{~mm})=200 *\left[0,5 *\left(\frac{8+38}{100}\right)+\frac{16+19+21+27}{100}\right] \\
h(\mathrm{~mm})=200 * 1,06 \\
h(\mathrm{~mm})=212
\end{gathered}
$$

Utilizando la ecuación 3 tenemos para conocer el total de agua que se encuentra en una hectárea a 1,0 m de profundidad tenemos:

$$
\begin{gathered}
h\left(\frac{m 3}{h a}\right)=212 * 10 \\
h\left(\frac{m 3}{h a}\right)=2120
\end{gathered}
$$




\section{Metodología 4}

Estimación de la humedad del suelo con contenido de agua no saturado en su perfil dividido en segmentos iguales en su longitud $(\Delta Z)$ y su respectiva humedad volumétrica $(\theta v)$ para cada segmento, donde se comienza a medir la humedad del suelo a partir de la superficie del mismo $(\theta 0)$ y luego continuando la medición a la longitud definida del segmento $(\Delta Z)$; tomando el promedio entre la humedad superficial y la humedad al primer $\Delta Z$ para el primer $\Delta Z / 2$.

Tomando en cuenta la figura 4, la fórmula para estimar el agua que se encuentra almacenada en el suelo en un momento dado se demuestra de la siguiente manera:

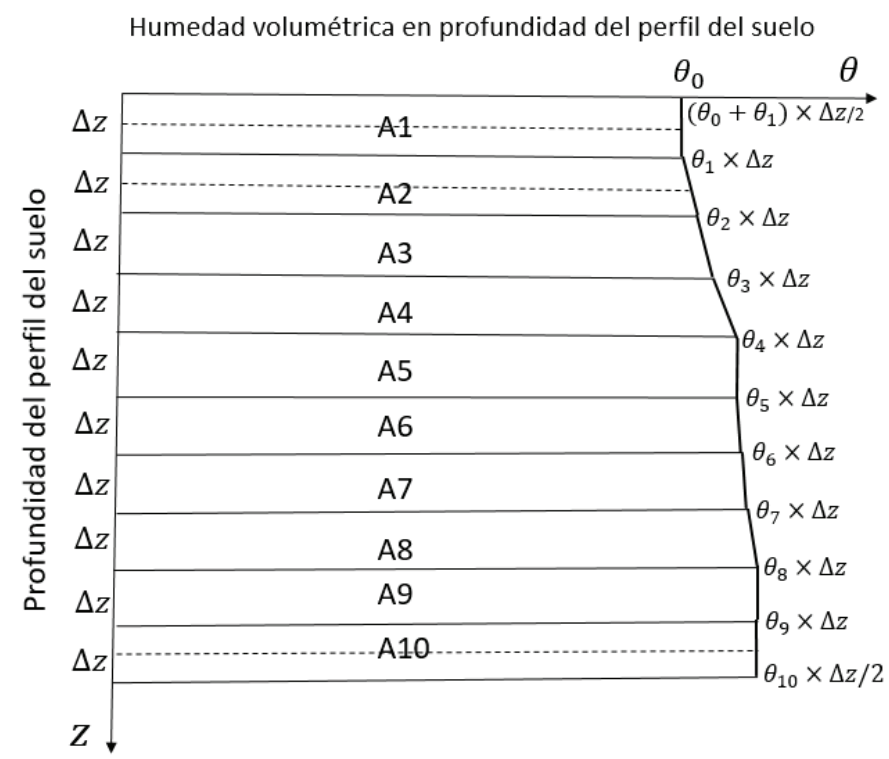

Figura 4. Estimación de la humedad del suelo realizando un promedio entre la primera medida y la segunda medida en profundidad a partir de la superficie del suelo $(\Delta z=0)$ para el primer $\Delta z / 2$ y luego cada $\Delta z$ en profundidad.

$$
\begin{gathered}
h(m m)=\frac{\Delta z}{2} \times \frac{\left(\theta_{0}+\theta_{1}\right)}{2}+\Delta z \theta_{1}+\Delta z \theta_{2}+\Delta z \theta_{3}+\Delta z \theta_{4}+\Delta z \theta_{5}+\Delta z \theta_{6}+\Delta z \theta_{7}+\Delta z \theta_{8} \\
\quad+\Delta z \theta_{9}+\frac{\Delta z}{2} * \theta_{10} \\
h(m m)=\Delta z\left(\frac{\left(\theta_{0}+\theta_{1}\right)}{4}+\theta_{1}+\theta_{2}+\theta_{3}+\theta_{4}+\theta_{5}+\theta_{6}+\theta_{7}+\theta_{8}+\theta_{9}+0,5 * \theta_{10}\right) \\
h(m m)=\Delta z\left(0,25 \theta_{0}+0,25 \theta_{1}+\theta_{1}+\theta_{2}+\theta_{3}+\theta_{4}+\theta_{5}+\theta_{6}+\theta_{7}+\theta_{8}+\theta_{9}+0,5 * \theta_{10}\right) \\
h(m m)=\Delta z\left(0,25 \theta_{0}+1,25 \theta_{1}+\theta_{2}+\theta_{3}+\theta_{4}+\theta_{5}+\theta_{6}+\theta_{7}+\theta_{8}+\theta_{9}+0,5 * \theta_{10}\right)
\end{gathered}
$$


ec. 13:

$$
h(m m)=\Delta z\left(0,25 \theta_{0}+1,25 \theta_{1}+0,5 * \theta_{n}+\sum_{i=2}^{n-1} \theta_{i}\right)
$$

y para el caso donde la humedad volumétrica esté expresada en porcentaje tenemos la ecuación 14

ec. 14:

$$
h(m m)=\Delta z\left(0,25 * \frac{\% \theta_{0}}{100}+1,25 * \frac{\% \theta_{1}}{100}+0,5 * \frac{\% \theta_{n}}{100}+\sum_{i=2}^{n-1} \frac{\left.\% \theta_{i}\right)}{100}\right)
$$

donde:

$\Delta Z$ = longitud del segmento de profundidad del suelo $(\mathrm{mm})$

$n=$ número total de capas en el perfil del suelo

$\theta o=$ humedad del suelo volumétrica superficial dada en proporción con base a la unidad

$\theta 1$ = humedad del suelo volumétrica medida al primer $\Delta Z$ de profundidad dada en proporción con base a la unidad

$\theta i=$ humedad del suelo volumétrica dada en proporción con base a la unidad, que se presenta en cada uno de los segmentos

$\theta n=$ humedad del suelo volumétrica dada en proporción con base a la unidad, que se presenta en el último segmento

$\% \theta 0$ = porcentaje de humedad volumétrica del suelo que se presenta a nivel superficial $\% \theta 1$ = porcentaje de humedad volumétrica del suelo medida a la profundidad del primer $\Delta Z$ $\% \theta i=$ porcentaje de humedad volumétrica del suelo que se presenta en cada uno de los segmentos

$\% \theta n=$ porcentaje de humedad volumétrica del suelo que se presenta en el último segmento

Ejemplo 4: Se tiene un perfil de suelo de 1,0 m de profundidad al cual se le hicieron muestreos para medir la humedad que hay en él. Para ello se dividió el perfil en capas de $20 \mathrm{~cm}$ donde cada una representa cada $\Delta z$ en profundidad y la humedad volumétrica se midió a partir de la superficie del suelo y luego se continuó con las medidas de humedad cada $20 \mathrm{~cm}$. Se toma la información del cuadro 3. Calcular el total de la humedad retenida en el perfil del suelo utilizando la metodología 4.

Utilizando la ecuación 14 tenemos:

$$
\begin{gathered}
h(\mathrm{~mm})=\Delta z\left(0,25 * \frac{\% \theta_{0}}{100}+1,25 * \frac{\% \theta_{1}}{100}+0,5 * \frac{\% \theta_{n}}{100}+\sum_{i=2}^{n-1} \frac{\% \theta_{i}}{100}\right) \\
h(\mathrm{~mm})=200\left(0,25 * \frac{8}{100}+1,25 * \frac{16}{100}+0,5 * \frac{38}{100}+\frac{19+21+27}{100}\right)
\end{gathered}
$$




$$
\begin{gathered}
h(\mathrm{~mm})=200 * 1,08 \\
h(\mathrm{~mm})=216
\end{gathered}
$$

Utilizando la ecuación 3 tenemos para conocer el total de agua que se encuentra en una hectárea a 1,0 m de profundidad tenemos:

$$
\begin{gathered}
h\left(\frac{m 3}{h a}\right)=216 * 10 \\
h\left(\frac{m 3}{h a}\right)=2160
\end{gathered}
$$

\section{Metodología 5}

Estimación de la humedad del suelo con contenido de agua no saturado en su perfil dividido en segmentos iguales en su longitud $(\Delta Z)$ y la respectiva humedad volumétrica $(\theta v)$ para cada segmento, donde se comienza a medir la humedad a partir de la superficie del suelo ( $\theta 0)$ y luego continuando la medición a la longitud definida del segmento $(\Delta Z)$; tomando el promedio entre las humedades superior e inferior de cada segmento $\Delta Z$.

Tomando en cuenta la figura 5, la fórmula para estimar el agua que se encuentra almacenada en el suelo en un momento dado se demuestra de la siguiente manera:

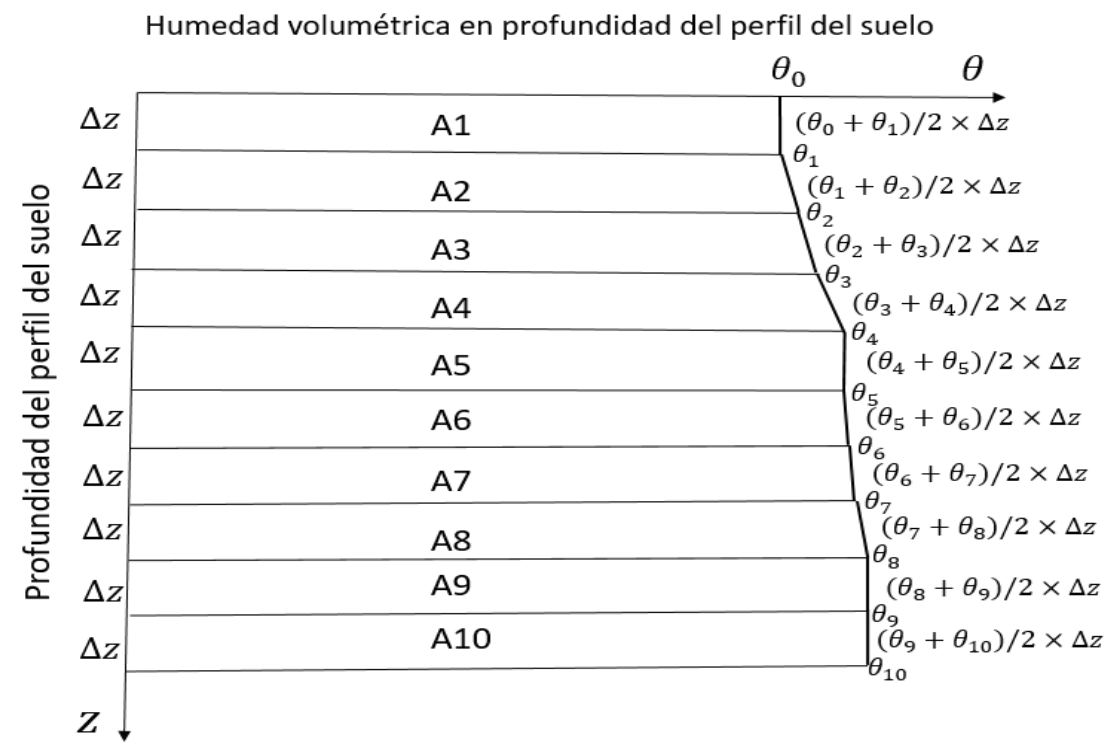

Figura 5. Estimación de la humedad del suelo realizando un promedio entre las humedades superior e inferior de cada segmento $\Delta Z$ 


$$
\begin{aligned}
& h(m m)=\Delta Z * \frac{(\theta 0+\theta 1)}{2}+\Delta Z * \frac{(\theta 1+\theta 2)}{2}+\Delta Z * \frac{(\theta 2+\theta 3)}{2}+\Delta Z * \frac{(\theta 3+\theta 4)}{2}+\Delta Z \\
& * \frac{(\theta 4+\theta 5)}{2}+\Delta Z * \frac{(\theta 5+\theta 6)}{2}+\Delta Z * \frac{(\theta 6+\theta 7)}{2}+\Delta Z * \frac{(\theta 7+\theta 8)}{2}+\Delta Z \\
& * \frac{(\theta 8+\theta 9)}{2}+\Delta Z * \frac{(\theta 9+\theta 10)}{2} \\
& h(m m)=\frac{\Delta Z}{2} *(\theta 0+\theta 1+\theta 1+\theta 2+\theta 2+\theta 3+\theta 3+\theta 4+\theta 4+\theta 5+\theta 5+\theta 6+\theta 6+\theta 7 \\
& +\theta 7+\theta 8+\theta 8+\theta 9++\theta 9+\theta 10) \\
& h(m m)=0,5 * \Delta Z *(\theta 0+\theta 1+\theta 1+\theta 2+\theta 2+\theta 3+\theta 3+\theta 4+\theta 4+\theta 5+\theta 5+\theta 6+\theta 6 \\
& +\theta 7+\theta 7+\theta 8+\theta 8+\theta 9++\theta 9+\theta 10) \\
& h(m m)=0,5 * \Delta Z *(\theta 0+2 * \theta 1+2 * \theta 2+2 * \theta 3+2 * \theta 4+2 * \theta 5+2 * \theta 6+2 * \theta 7+2 \\
& * \theta 8+2 * \theta 9+\theta 10) \\
& h(m m)=0,5 * \Delta Z *(\theta 0+\theta 10+2 *(\theta 1+\theta 2+\theta 3+\theta 4+\theta 5+\theta 6+\theta 7+\theta 8+\theta 9)) \\
& h(m m)=0,5 * \Delta Z *\left[\theta 0+\theta 10+2 *\left(\sum_{i=1}^{n-1} \theta i\right)\right.
\end{aligned}
$$

de donde sustituyendo 10 por n se puede deducir la ecuación 15.

ec. 15:

$$
\left.h(m m)=0,5 * \Delta Z *\left[\theta o+\theta n+2 *\left(\sum_{i=1}^{n-1} \theta i\right)\right)\right]
$$

y para el caso en que la humedad volumétrica se encuentre en términos de porcentaje tenemos la ecuación 16:

ec. 16:

$$
h(m m)=0,5 * \Delta Z *\left[\frac{\% \theta 0}{100}+\frac{\% \theta n}{100}+2 *\left(\sum_{i=1}^{n-1} \frac{\% \theta i}{100}\right)\right]
$$

donde:

$\Delta Z=$ longitud del segmento de profundidad del suelo $(\mathrm{mm})$

$n=$ número total de capas en el perfil del suelo

$\theta 0$ = humedad del suelo volumétrica superficial dada en proporción con base a la unidad

$\theta i=$ humedad del suelo volumétrica dada en proporción con base a la unidad, que se presenta en cada uno de los segmentos 
$\theta n=$ humedad del suelo volumétrica dada en proporción con base a la unidad, que se presenta en el último segmento

$\% \theta 0=$ porcentaje de humedad volumétrica del suelo a nivel superficial dada en proporción con base a la unidad

$\% \theta i=$ porcentaje de humedad volumétrica del suelo que se presenta en cada uno de los segmentos

$\% \theta n=$ porcentaje de humedad volumétrica del suelo que se presenta en el último segmento

Para expresar dicha humedad en metros cúbicos por cada hectárea se puede utilizar la siguiente ecuación 17:

ec. 17:

$$
h\left(\frac{m 3}{h a}\right)=\left(0,5 * \Delta Z *\left[\theta 0+\theta n+2 *\left(\sum_{i=1}^{n-1} \theta i\right)\right]\right) * 10
$$

y para el caso en que la humedad volumétrica se encuentre en términos de porcentaje tenemos la ecuación 18:

ec. 18:

$$
h\left(\frac{m 3}{h a}\right)=\left(0,5 * \Delta Z *\left[\frac{\% \theta 0}{100}+\frac{\% \theta n}{100}+2 *\left(\sum_{i=1}^{n-1} \frac{\% \theta i}{100}\right)\right]\right) * 10
$$

Ejemplo 5: Se tiene un perfil de suelo de 1,0 m de profundidad al cual se le hicieron muestreos para medir la humedad que hay en él. Para ello se dividió el perfil en capas de $20 \mathrm{~cm}$ donde cada una representa cada $\Delta z$ en profundidad y la humedad volumétrica se midió a partir de la superficie del suelo y luego se continuó con las medidas de humedad cada $20 \mathrm{~cm}$ o cada segmento $\Delta z$. Se toma la información del cuadro 3. Calcular el total de la humedad retenida en el perfil del suelo según metodología 5.

Utilizando la ecuación 16 tenemos lo siguiente:

$$
\begin{gathered}
h(\mathrm{~mm})=0,5 * \Delta Z *\left[\frac{\% \theta 0}{100}+\frac{\% \theta n}{100}+2 *\left(\sum_{i=1}^{n-1} \frac{\% \theta i}{100}\right)\right] \\
h(\mathrm{~mm})=0,5 * 200 *\left[\frac{8}{100}+\frac{38}{100}+2 *\left(\frac{16+19+21+27}{100}\right)\right] \\
h=0,5 * 200 *(2,12) \\
h(\mathrm{~mm})=212
\end{gathered}
$$

Utilizando la ecuación 3 tenemos para conocer el total de agua que se encuentra en una hectárea a 1,0 m de profundidad tenemos: 


$$
\begin{gathered}
h\left(\frac{m 3}{h a}\right)=212 * 10 \\
h\left(\frac{m 3}{h a}\right)=2120
\end{gathered}
$$

Cuadro 4. Estimación de la cantidad de metros cúbicos por hectárea a 1 metro de profundidad para cada metodología propuesta.

\begin{tabular}{|c|c|}
\hline Metodología & Volumen( m3/ha) \\
\hline 1 & 2420 \\
\hline 2 & 2200 \\
\hline 3 & 2120 \\
\hline 4 & 2160 \\
\hline 5 & 2120 \\
\hline Promedio $=$ & 2204,00 \\
\hline Desviación Estandar $=$ & 125,22 \\
\hline \% Coef. Variación $=$ & 5,68 \\
\hline
\end{tabular}

\section{Conclusiones}

Las ecuaciones demostradas por la metodología del Trapecio muestran con respecto a todos los resultados de los ejemplos realizados que varían como máximo un 13,60\% en promedio que viene a representar $300 \mathrm{~m}^{3} / \mathrm{ha}$ y presentan un coeficiente de variación del 5,68\%. La variación en la estimación cuando no se mide la humedad superficial es del 9,52\% y la variación cuando se mide la humedad superficial es de 1,88\%. De aquí se puede derivar la importancia en la estimación de la medida de la humedad del suelo en la superficie del suelo la cual aumenta la precisión de las estimaciones obtenidos.

Se puede observar que dependiendo de cómo se realiza el muestreo en la profundidad del suelo y de cómo se definan las humedades para los segmentos, se van a presentar diferencias en las estimaciones del contenido de humedad en un perfil de suelo.

De los ejemplos de estimación por las diferentes metodologías podemos observar que las metodologías 3 y 5 dan resultados iguales y a su vez, las metodologías 1, 2 y 4 presentan diferentes resultados entre ellos y diferentes resultados con las 3 y 5 . Además, las metodologías que presentan menores valores son las 3 y 5 y la que presenta el mayor valor de estimación es la metodología 1.

Las metodologías con mayor precisión respecto a las otras son las 3 y 5 debido a dos condiciones conjuntas que son menor variación y la medida de la humedad superficial.

\section{Referencias}

[1] Organizción de las Naciones Unidas para la Alimentación y la Agricultura (FAO), Roma, 2015, p. 4. Obtenido desde http://www.fao.org/fileadmin/user_upload/soils-2015/docs/Fact_sheets/print_IYS_Water_es.pdf

[2] G. Angella, C. Frías y R. Salgado, 2016. Obtenido desde https://www.researchgate.net/publication/322888562_ Conceptos_basicos_de_las_relaciones_agua-suelo-planta

[3] R. López Olivari, Boletín INIA No 340: Manejo y uso eficiente del agua de riego intrapredial para el sur de chile, Temuco, 2016, p. 497. Obtenido desde https://puntoganadero.cl/imagenes/upload/_5cc083a547bfb.pdf 
[4] Organización de las Naciones Unidas para la Alimetación y la Agricultura (FAO), Santiago, 2013. Obtenido desde http://www.fao.org/3/i3247s/i3247s.pdf

[5] P. L. Libardi, Dinâmica da Água do Solo, 2da ed., São Paulo, 2012, p. 350.

[6] V. da Silva, P. de Azevedo, B. da Silva, L. Bassoi, A. Teixeira, J. Soares y J. e Silva, «Estimativa da evapotranspiração da mangueira com base no balanço hídrico do solo,» Revista Brasileira de Engenharia Agricola e Ambiental, vol. 5, nº 3, pp. 456 - 462, 2001. Obtenido desde https://www.researchgate.net/publication/250985336_Estimativa_da_evapotranspiracao_da_mangueira_com_base_no_balanco_hidrico_do_solo

[7] V. da Silva, P. de Azevedo, B. da Silva, L. Bassoi, A. de Castro, J. Soares y J. Sobrinho, Campina Grande, 2000, pp. 240 - 249. Obtenido desde https://www.alice.cnptia.embrapa.br/bitstream/doc/134371/1/OPB276. pdf

[8] d. S. Cícero, A. de Andrade, J. de S. Lima, A. Antonimo, E. de Souza y I. da Silva, «Balanço hídrico da cultura da mamona sob condições de sequeiro, » Revista BrasileiraEngenharia Agrícola e Ambiental, vol. 17, n 1, pp. 3 - 10, 2013. Obtenido desde

[9] Organismo Internacional de Energía Atómica, Colección de cursos de capacitación No 16/S, Viena, 2003. Obtenido desde

[10] R. Haverkamp, M. Vauclin y G. Vachaud, «Error analysis in estimating soil water content from neutron probe measurements: 1. Local standpoint,» Soil Science, vol. 137, n² 2, pp. 78 - 90, Febrero 1984. Obtenido desde https://journals.Iww.com/soilsci/Abstract/1984/02000/Error_Analysis_in_Estimating_Soil_Water_Content.2.aspx

[11] A. E. Chavarría Vidal, Apuntes de clases de Relación Suelo Agua, Cartago, Cartago, 2012, p. 203. 